\title{
EXPONENTIAL GROWTH RATE FOR DERIVATIVES OF STOCHASTIC FLOWS
}

\author{
HOLGER VAN BARGEN*, MICHAEL SCHEUTZOW ${ }^{\dagger}$ \\ SIMON WASSERROTH ${ }^{\ddagger}$ \\ Institut für Mathematik, MA 7-5, Technische Universität Berlin \\ Straße des 17. Juni 136, 10623 Berlin, Germany \\ *van_barg@math.tu-berlin.de \\ ${ }^{\dagger}$ ms@math.tu-berlin.de \\ ${ }^{\ddagger}$ wasserroth@math.tu-berlin.de
}

\begin{abstract}
We show that for a large class of stochastic flows the spatial derivative grows at most exponentially fast even if one takes the supremum over a bounded set of initial points. We derive explicit bounds on the growth rates that depend on the local characteristics of the flow and the box dimension of the set.
\end{abstract}

Keywords. stochastic flows, stochastic differential equations, spatial derivatives, exponential growth

AMS Subject Classification: 60H10, 60F15, 60G17, 60G60, 37H15

\section{Introduction and Set-Up}

The evolution of the diameter of a bounded set under the action of a stochastic flow has been studied since the 1990's (see [3], [4], [5], [8], [9], [12], and the survey article [11] to name just a few references). For a large class of flows - including isotropic Brownian flows (IBFs) with non-negative top Lyapunov exponent - the diameter is known to be linearly growing in time. In this paper, we will consider the evolution of the spatial derivative of a flow in time and derive an explicit upper bound on the supremum of the spatial derivative taken over a bounded set. Our bound depends on the box dimension of the set. In the case of IBFs such a result has been obtained in [13] with a different (and more technical) proof. We will be much more general with our set-up but, contrary to [13], will not derive lower bounds for the growth rates.

We point out that there is a close link between this paper and work of Peter Imkeller: in [6], the growth of the spatial derivative of a flow in the spatial direction was studied over a fixed time horizon $[0, T]$ and we did not care about constants (even $T$ was regarded as a constant). Still, the proof of Lemma 3.1, which constitutes the core of our results, largely follows that of Proposition 2.3 of [6]. Apart from keeping track 
of constants, our proof here differs from that in [6] towards the end when we apply a non-linear Gronwall-type Lemma (the usual Gronwall Lemma will not provide an exponential growth rate in $T$ ).

Exponential bounds on the growth of spatial derivatives play an important role in the proof of Pesin's formula for stochastic flows (see [10]). They can also be used to obtain bounds on the exponential growth rate of e.g. the length of a curve under a flow. Even though we try to keep track of constants, we make no claims about optimality (and we conjecture that our bound is far from optimal). We give explicit formulas for the exponential growth rate only for the first order derivative but indicate how such bounds can be obtained also for higher order derivatives under additional smoothness assumptions.

The paper is organized as follows: we start by defining a suitable class of stochastic flows. Then, we provide a general result - Theorem 2.2 - which shows how one can obtain exponential growth rates for a random field $\psi$ indexed by $\mathbf{R}^{d}$ given moment bounds on the field and on two-point differences of the field. Afterwards, we apply this theorem to the derivative of a stochastic flow. Here, the main task is to compute the moment bounds needed in order to apply Theorem 2.2. Then, we specialize to IBFs. [7].

Let us introduce our set-up which is essentially the same as in [6] and is based on

Let $F(x, t), t \geq 0$ be a family of $\mathbf{R}^{d}$-valued continuous semimartingales on a filtered probability space $\left(\Omega, \mathcal{F},\left(\mathcal{F}_{t}\right)_{t \geq 0}, \mathbf{P}\right)$ indexed by $x \in \mathbf{R}^{d}$, starting at 0 . Let $F(x, t)=M(x, t)+V(x, t)$ be the canonical decomposition into a local martingale $M$ and a process $V$ of locally bounded variation (both starting at 0 ). We will assume throughout that both $M$ and $V$ are jointly continuous in $(x, t)$. Furthermore we assume that there exist $a: \mathbf{R}^{d} \times \mathbf{R}^{d} \times\left[0, \infty\left[\times \Omega \rightarrow \mathbf{R}^{d \times d}\right.\right.$ which is continuous in the first two and predictable in the last two variables and $b: \mathbf{R}^{d} \times\left[0, \infty\left[\times \Omega \rightarrow \mathbf{R}^{d}\right.\right.$ which is continuous in the first and predictable in the last two variables such that

$$
\left\langle M_{i}(x, .), M_{j}(y, .)\right\rangle(t)=\int_{0}^{t} a_{i j}(x, y, u) \mathrm{d} u, \quad V_{i}(x, t)=\int_{0}^{t} b_{i}(x, u) \mathrm{d} u .
$$

Here, $\langle.,$.$\rangle denotes the joint quadratic variation. The pair of random fields (a, b)$ is called the local characteristics of the semimartingale field $F$. We will abbreviate $\mathcal{A}(x, y, t):=a(x, x, t)-a(x, y, t)-a(y, x, t)+a(y, y, t)$ (which is the derivative of the quadratic variation of $M(x, t)-M(y, t))$. Throughout, we will assume that the following hypothesis holds: 


\section{Hypothesis (A):}

$$
\begin{aligned}
& \underset{\omega \in \Omega}{\operatorname{esssup}} \sup _{t \geq 0} \sup _{x, y \in \mathbf{R}^{d}}\left(\frac{\|a(x, y, t)\|}{(1+|x|)(1+|y|)}+\sum_{k=1}^{d}\left\|D_{x_{k}} D_{y_{k}} a(x, y, t)\right\|\right. \\
& \left.+\sum_{k=1}^{d}\left\|D_{x_{k}} D_{y_{k}} a(., ., t)\right\|^{\sim}\right)<\infty, \text { and } \\
& \underset{\omega \in \Omega}{\operatorname{essup} \sup _{t \geq 0}}\left(\sup _{x \in \mathbf{R}^{d}} \frac{|b(x, t)|}{1+|x|}+\sup _{x \in \mathbf{R}^{d}}\left\|D_{x} b(x, t)\right\|+\sup _{x \neq y \in \mathbf{R}^{d}} \frac{\left\|D_{x} b(x, t)-D_{y} b(y, t)\right\|}{|x-y|}\right)<\infty,
\end{aligned}
$$

where

$$
\|f(., .)\|^{\sim}:=\sup _{x \neq x^{\prime}, y \neq y^{\prime}}\left\{\frac{\left\|f(x, y)-f\left(x^{\prime}, y\right)-f\left(x, y^{\prime}\right)+f\left(x^{\prime}, y^{\prime}\right)\right\|}{\left|x-x^{\prime}\right|\left|y-y^{\prime}\right|}\right\} .
$$

Since Hypothesis (A) implies the assumptions of [7, Theorem 4.6.5] (with $k=1$, $\delta=1$ ), the stochastic differential equation

$$
\mathrm{d} X(t)=F(X(t), \mathrm{d} t), \quad X(s)=x, t \geq s
$$

not only admits a unique solution for each fixed $x \in \mathbf{R}^{d}$ and $s \geq 0$, but even generates a stochastic flow of diffeomorphisms $\phi$, i.e. there exist a random field $\phi:[0, \infty)^{2} \times$ $\mathbf{R}^{d} \times \Omega \rightarrow \mathbf{R}^{d}$ and a set $\Omega_{0}$ of full measure such that

- $t \mapsto \phi_{s, t}(x), t \geq s$ solves (1.1) for all $x \in \mathbf{R}^{d}, s \geq 0$.

- $\phi_{s, t}(\omega)$ is a diffeomorphism on $\mathbf{R}^{d}$ for all $s, t \geq 0, \omega \in \Omega_{0}$.

- $\phi_{s, u}=\phi_{t, u} \circ \phi_{s, t}$ for all $s, t, u \geq 0, \omega \in \Omega_{0}$.

- $(s, t, u) \mapsto \phi_{s, t}(x)$ is continuous for all $\omega \in \Omega_{0}$.

We will often write $x_{t}:=\phi_{t}(x):=\phi_{0, t}(x, \omega)$.

\section{Exponential Growth Rates: General Results}

In the following lemma and theorem, $o(T)$ stands for a function $g(T)$ which may depend on $q$, but not on $x, y$ such that $\lim _{T \rightarrow \infty} g(T) / T=0$.

Lemma 2.1. Let $(E, \rho)$ be a complete, separable metric space and let $(t, x) \mapsto \psi_{t}(x)$ be a continuous random field on $[0, \infty) \times \mathbf{R}^{d}$ with values in $(E, \rho)$ which satisfies

$$
\mathbf{E} \sup _{0 \leq t \leq T} \rho\left(\psi_{t}(x), \psi_{t}(y)\right)^{q} \leq|x-y|^{q} \exp \left\{\left(c q^{2}+\hat{c} q\right) T+o(T)\right\}
$$

for some $q>d$ and all $x, y \in \mathbf{R}^{d}$. Then, for $u>0$, we have

$$
\mathbf{P}\left\{\sup _{x, y \in[0,1]^{d}} \sup _{0 \leq t \leq T} \rho\left(\psi_{t}(x), \psi_{t}(y)\right) \geq u\right\} \leq \exp \left\{\left(c q^{2}+\hat{c} q\right) T+o(T)\right\} u^{-q},
$$


Proof. This follows from Kolmogorov's continuity theorem (e.g. in the version of [11, Lemma 2.1]).

Theorem 2.2. Let $(E,\|\|$.$) be a separable real Banach space and let (t, x) \mapsto \psi_{t}(x)$ be a continuous random field on $[0, \infty) \times \mathbf{R}^{d}$ with values in $(E,\|\|$.$) which satisfies$

$$
\mathbf{E} \sup _{0 \leq t \leq T}\left\|\psi_{t}(x)-\psi_{t}(y)\right\|^{q} \leq|x-y|^{q} \exp \left\{\left(c q^{2}+\hat{c} q\right) T+o(T)\right\}
$$

for some $c>0, \hat{c} \in \mathbf{R}$, all $q>d$ and all $x, y \in \mathbf{R}^{d}$. Assume further that

$$
\sup _{x} \mathbf{E} \sup _{0 \leq t \leq T}\left\|\psi_{t}(x)\right\|^{q} \leq \exp \left\{\left(k q^{2}+\hat{k} q\right) T+o(T)\right\}
$$

for some $k>0, \hat{k} \in \mathbf{R}$ and for all $q \geq 0$. If $\mathbb{X}$ is any compact subset of $\mathbf{R}^{d}$ with box dimension $\Delta$, then

$$
\limsup _{T \rightarrow \infty} \frac{1}{T} \log \sup _{x \in \mathbb{X}} \sup _{0 \leq t \leq T}\left\|\psi_{t}(x)\right\| \leq \xi \text { a.s. }
$$

where

$$
\xi= \begin{cases}\hat{k} & \text { if } \hat{k} \geq c d+\hat{c} \\ \hat{k}+2 \sqrt{k \Delta \gamma_{1}} & \text { if } \hat{k} \leq c d+\hat{c} \text { and } 2 \sqrt{c k} \Delta d+c d^{2}-2 c \Delta d+\Delta(\hat{k}-\hat{c}) \geq 0 \\ \hat{k}+2 \sqrt{k \Delta \gamma_{2}} & \text { if } \hat{k} \leq c d+\hat{c} \text { and } 2 \sqrt{c k} \Delta d+c d^{2}-2 c \Delta d+\Delta(\hat{k}-\hat{c}) \leq 0\end{cases}
$$

where

$$
\begin{aligned}
& \gamma_{1}:= \begin{cases}\frac{c d+\hat{c}-\hat{k}}{2 \sqrt{k d}} & \text { if } \Delta=d \\
\left(\left(1-\frac{\Delta}{d}\right)^{-1}\left(-\sqrt{k \Delta}+\sqrt{k \Delta+\left(1-\frac{\Delta}{d}\right)(c d+\hat{c}-\hat{k})}\right)\right)^{2} & \text { if } \Delta<d\end{cases} \\
& \gamma_{2}:=\left(\sqrt{c \Delta}+\sqrt{k \Delta}+\sqrt{(\sqrt{c}-\sqrt{k})^{2} \Delta-\hat{k}+\hat{c}}\right)^{2} .
\end{aligned}
$$

Proof. Let $\varepsilon>0$. For each $\gamma>0$, we can cover the set $\mathbb{X}$ with $N \leq \mathrm{e}^{\gamma T(\Delta+\varepsilon)}$ balls of diameter $\mathrm{e}^{-\gamma T}$ in case $T$ is large enough. For given such $\gamma, T$ we denote these balls by $\mathbb{X}_{1}, \ldots \mathbb{X}_{N}$ and their centers by $x_{1}, \ldots, x_{N}$. Let $r>0$. Then, using Lemma 2.1, we obtain

$\mathbf{P}\left\{\exists i \in\{1, \ldots, N\}\right.$ s.t. $\left.\sup _{0 \leq t \leq T} \operatorname{diam}\left(\psi_{t}\left(\mathbb{X}_{i}\right)\right) \geq \mathrm{e}^{r T}\right\} \leq \mathrm{e}^{\gamma T(\Delta+\varepsilon)} \mathrm{e}^{\left(c q^{2}+(\hat{c}-\gamma-r) q\right) T+o(T)}$,

and therefore

$$
\begin{aligned}
B(r) & :=\limsup _{T \rightarrow \infty} \frac{1}{T} \log \mathbf{P}\left\{\exists i \in\{1, \ldots, N\} \text { s.t. } \sup _{0 \leq t \leq T} \operatorname{diam}\left(\psi_{t}\left(\mathbb{X}_{i}\right)\right) \geq \mathrm{e}^{r T}\right\} \\
& \leq \gamma \Delta+c q^{2}+(\hat{c}-\gamma-r) q .
\end{aligned}
$$

Optimizing over $q>d$ yields

$$
B(r) \leq\left\{\begin{array}{cc}
\gamma \Delta-\frac{(r-\hat{c}+\gamma)^{2}}{4 c} & \text { if } r \geq 2 c d+\hat{c}-\gamma \\
\gamma \Delta+(c d+\hat{c}-\gamma-r) d & \text { if } r<2 c d+\hat{c}-\gamma
\end{array}\right.
$$


Further,

$$
\mathbf{P}\left\{\exists i \in\{1, \ldots, N\} \text { s.t. } \sup _{0 \leq t \leq T}\left\|\psi_{t}\left(x_{i}\right)\right\| \geq \mathrm{e}^{r T}\right\} \leq \mathrm{e}^{\gamma T(\Delta+\varepsilon)} \mathrm{e}^{\left(k q^{2}+(\hat{k}-r) q+o(1)\right) T}
$$

and therefore

$C(r):=\limsup _{T \rightarrow \infty} \frac{1}{T} \log \mathbf{P}\left\{\exists i \in\{1, \ldots, N\}\right.$ s.t. $\left.\sup _{0 \leq t \leq T}\left\|\psi_{t}\left(x_{i}\right)\right\| \geq \mathrm{e}^{r T}\right\} \leq \gamma \Delta+k q^{2}+(\hat{k}-r) q$.

Optimizing over $q \geq 0$, we get

$$
C(r) \leq \gamma \Delta-\frac{(r-\hat{k})^{2}}{4 k}
$$

provided that $r \geq \hat{k}$ which we will assume to hold from now on.

Once we know that for a particular value of $r>0$

$$
A(r):=\limsup _{T \rightarrow \infty} \frac{1}{T} \log \mathbf{P}\left\{\sup _{0 \leq t \leq T} \sup _{x \in \mathbb{X}}\left\|\psi_{t}(x)\right\| \geq 2 e^{r T}\right\}<0,
$$

then a simple Borel-Cantelli argument (using the fact that $T \mapsto \sup _{0 \leq t \leq T} \sup _{x \in \mathbb{X}}\left\|\psi_{t}(x)\right\|$ is non-decreasing) shows that (2.4) holds with $\xi$ replaced by $r$. Since $A(r) \leq B(r) \vee$ $C(r)$, we have $A(r)<0$ whenever there exists some $\gamma>0$ such that both upper bounds of $B(r)$ and $C(r)$ are negative. Defining $\xi$ as the infimum over all such $r$, we obtain

$$
\xi=\inf _{\gamma>0}\left((\hat{k}+2 \sqrt{k \gamma \Delta}) \vee\left\{\begin{array}{cc}
2 \sqrt{c \gamma \Delta}+\hat{c}-\gamma & : \gamma \Delta \geq c d^{2} \\
\gamma \Delta d^{-1}+c d+\hat{c}-\gamma & : \gamma \Delta \leq c d^{2}
\end{array}\right) .\right.
$$

Computing the infimum, we obtain the result in the theorem.

Remark 2.3. It follows from (2.5) that

$$
\hat{k} \leq \xi \leq(c d+\hat{c}) \vee \hat{k} .
$$

Note that the lower bound is attained in case $\Delta=0$.

Remark 2.4. Our assumptions on the range of admissible values of $q$ in Theorem 2.2 are a bit arbitrary (but motivated by applications to IBFs). It is clear from the proof that (2.4) still holds with a larger value of $\xi$ if we only assume that (2.2) holds for some $q>d$ and that (2.3) holds for some $q>0$ (the values of $q$ can be different).

\section{Application to the Derivative of a Stochastic Flow}

Next, we want to use the results in the previous section to obtain bounds on the exponential growth rate of the supremum of the derivative of a stochastic flow taken over all initial points in a compact set of box dimension $\Delta$. In order to apply Theorem 2.2 , we have to estimate moments of the difference of derivatives of a stochastic flow. We 
start by introducing some more notation (as in [6]).

Let $\phi=\left(\phi^{1}, \ldots, \phi^{d}\right)$ be a stochastic flow of diffeomorphisms generated by (1.1) satisfying Hypothesis (A) and let $p \geq 1$. Fix $i \in\{1, \ldots, d\}$ and define $Y_{j}(t):=$ $D_{i} \phi_{t}^{j}(x), Z(t):=\sup _{0 \leq s \leq t}\left(\sum_{j=1}^{d} Y_{j}^{2}(s)\right)^{1 / 2}, f_{p}(t)=\max _{i}\left(\mathbf{E} Z(t)^{p}\right)^{1 / p}$, and $\tilde{f}_{p}(t)=\max _{i}\left(\mathbf{E}\left(\sum_{j=1}^{d} Y_{j}^{2}(t)\right)^{p / 2}\right)^{1 / p}$. Here and in the following, we write $D_{k}$ instead of $D_{x_{k}}$ or $D_{y_{k}}$. We will also need two two-point versions $V_{j}(t):=D_{i} \phi_{0, t}^{j}(x)-$ $D_{i} \phi_{0, t}^{j}(y), W(t):=\sup _{0 \leq s \leq t}\left(\sum_{j=1}^{d} V_{j}^{2}(s)\right)^{1 / 2}$ and $g_{p}(t):=\max _{i}\left(\mathbf{E}\left(W^{p}(t)\right)^{1 / p}\right.$. Let $C_{p}$ denote the constant in Burkholder's inequality. It is well-known, that there exists a constant $k_{5}>0$ such that $C_{p} \leq\left(k_{5} p^{1 / 2}\right)^{p}$ for all $p \geq 2$ (see [1]) (one can choose $k_{5}=2 \sqrt{5}$ ). We point out that $D_{k} D_{k}$ in front of a function of two spatial arguments means that we differentiate with respect to the $k$-th component of both arguments. Note that we trivially have $\tilde{f}_{p}(t) \leq f_{p}(t)$, so the terms $\tilde{f}_{p}(t)$ which appear in the upper bound of $H$ in the following lemma can be replaced by $f_{p}(t)$ (but one may get better bounds by not doing this).

Lemma 3.1. Let $\phi$ be the flow generated by $F$ satisfying Hypothesis $(A)$ and denote

$$
\begin{aligned}
& \text { 1. } k_{1}:=\operatorname{esssup}_{\omega \in \Omega} \sup _{\bar{x} \in \mathbf{R}^{d}, t \geq 0,1 \leq j, k \leq d}\left|D_{k} D_{k} a_{j j}(\bar{x}, \bar{x}, t)\right|, \\
& \text { 2. } k_{2}:=\operatorname{essup}_{\omega \in \Omega} \sup _{\bar{x}, \bar{y} \in \mathbf{R}^{d}, t \geq 0,1 \leq j, k \leq d} \frac{\left|D_{k} D_{k} \mathcal{A}_{j j}(\bar{x}, \bar{y}, t)\right|}{|\bar{x}-\bar{y}|^{2}}, \\
& \text { 3. } k_{3}:=\operatorname{esssup}_{\omega \in \Omega} \sup _{\bar{x} \in \mathbf{R}^{d}, t \geq 0,1 \leq j, k \leq d}\left|D_{k} b_{j}(\bar{x}, t)\right|, \\
& \text { 4. } k_{4}:=\operatorname{essup}_{\omega \in \Omega} \sup _{\bar{x}, \bar{y} \in \mathbf{R}^{d}, t \geq 0,1 \leq j, k \leq d} \frac{\left|D_{k} b_{j}(\bar{x}, t)-D_{k} b_{j}(\bar{y}, t)\right|}{|\bar{x}-\bar{y}|} .
\end{aligned}
$$

Then there exist constants $\Lambda, \bar{c}$ and $\sigma \geq 0$ such that for all $p \geq 2, t \geq 0$ and $\bar{x}, \bar{y} \in \mathbf{R}^{d}$,

$$
\left(\mathbf{E}\left|\phi_{t}(\bar{x})-\phi_{t}(\bar{y})\right|^{p}\right)^{1 / p} \leq \bar{c}|\bar{x}-\bar{y}| \mathrm{e}^{\left(\Lambda+p \sigma^{2} / 2\right) t} .
$$

Further, for all $p \geq 2, x \in \mathbf{R}^{d}$, and all $\alpha_{1}, \alpha_{2}, \alpha_{3}>1$ whose reciprocals sum up to 1 , $f_{p}$ satisfies

$f_{p}^{2}(t) \leq \alpha_{1}+\alpha_{1} \frac{\alpha_{2} \bar{d}^{2} k_{1} C_{p}^{2 / p}+\sqrt{\alpha_{3}} \bar{d} k_{3}}{\alpha_{2} \bar{d}^{2} k_{1} C_{p}^{2 / p}+2 \sqrt{\alpha_{3}} \bar{d} k_{3}}\left(\exp \left\{\left(\alpha_{2} \bar{d}^{2} k_{1} C_{p}^{2 / p}+2 \sqrt{\alpha_{3}} \bar{d} k_{3}\right) t\right\}-1\right)$,

where $\bar{d}=d^{2-1 / p}$. Further, for all $p \geq 2, x, y \in \mathbf{R}^{d}$, and all $\beta_{m}>1, m=1,2,3,4$ whose reciprocals sum up to 1 , we have

$$
g_{p}^{2}(t) \leq H(t)+H(t) \frac{\bar{C}_{1}+\sqrt{\bar{C}_{2}}}{\bar{C}_{1}+2 \sqrt{\bar{C}_{2}}}\left(\exp \left\{\left(\bar{C}_{1}+2 \sqrt{\bar{C}_{2}}\right) t\right\}-1\right)
$$

where $\bar{C}_{1}:=\beta_{1} d^{3-2 / p} C_{p}^{2 / p} k_{1}, \bar{C}_{2}:=\beta_{3} d^{3-2 / p} k_{3}^{2}$ and

$H(t):=d^{3} \bar{c}^{2}|x-y|^{2}\left(C_{p}^{2 / p} \beta_{2} k_{2} \int_{0}^{t} \tilde{f}_{2 p}^{2}(s) \mathrm{e}^{2\left(\Lambda+p \sigma^{2}\right) s} \mathrm{~d} s+\beta_{4} k_{4}^{2}\left(\int_{0}^{t} \tilde{f}_{2 p}(s) \mathrm{e}^{\left(\Lambda+\sigma^{2} p\right) s} \mathrm{~d} s\right)^{2}\right)$. 
Proof. First note that $k_{1}, \ldots, k_{4}<\infty$ since $F$ satisfies Hypothesis (A). Assertion (3.6) follows from [11, Lemma 2.6] and the fact that $F$ satisfies (A) (one can choose $\sigma=\tilde{a}$ and $\Lambda=\tilde{b}+(d-1) \tilde{a}^{2} / 2$, where $\tilde{b}$ is a deterministic upper bound of the Lipschitz constant of $b$ and $\tilde{a} \geq 0$ is chosen such that $\|\mathcal{A}(x, y, t, \omega)\| \leq \tilde{a}^{2}|x-y|^{2}$ for all $x, y \in \mathbf{R}^{d}$ and almost all $\omega \in \Omega$ ). Fix $i \in\{1, \ldots, d\}$. We have

$$
Y_{j}(s)=\delta_{i, j}+\sum_{n=1}^{d} \int_{0}^{s} Y_{n}(u) D_{n} F_{j}\left(x_{u}, \mathrm{~d} u\right)
$$

(see [7, p.174 (21)] or [6, (18)]). Applying Burkholder's inequality, we get

$$
\begin{aligned}
\left(\mathbf{E} \sup _{0 \leq s \leq t}\left|Y_{j}(s)\right|^{p}\right)^{1 / p} \leq \delta_{i, j}+\sum_{n=1}^{d}\left(\mathbf{E}\left(\sup _{0 \leq s \leq t} \int_{0}^{s} Y_{n}(u) D_{n} F_{j}\left(x_{u}, \mathrm{~d} u\right)\right)^{p}\right)^{1 / p} \\
\leq \delta_{i, j}+C_{p}^{1 / p} \sum_{n=1}^{d}\left(\mathbf{E}\left(\int_{0}^{t} Y_{n}^{2}(u) D_{n} D_{n} a_{j j}\left(x_{u}, x_{u}, u\right) \mathrm{d} u\right)^{p / 2}\right)^{1 / p} \\
\quad+\sum_{n=1}^{d}\left(\mathbf{E}\left(\int_{0}^{t}\left|Y_{n}(u) D_{n} b_{j}\left(x_{u}, u\right)\right| \mathrm{d} u\right)^{p}\right)^{1 / p} \\
\leq \delta_{i, j}+C_{p}^{1 / p} \sqrt{k_{1}} \sum_{n=1}^{d}\left(\mathbf{E}\left(\int_{0}^{t} Y_{n}^{2}(u) \mathrm{d} u\right)^{p / 2}\right)^{1 / p} \\
\quad+k_{3} \sum_{n=1}^{d}\left(\mathbf{E}\left(\int_{0}^{t}\left|Y_{n}(u)\right| \mathrm{d} u\right)^{p}\right)^{1 / p} .
\end{aligned}
$$

Since $p \geq 2$, Jensen's inequality implies

$$
\begin{aligned}
\sum_{n=1}^{d} & \left(\mathbf{E}\left(\int_{0}^{t} Y_{n}^{2}(u) \mathrm{d} u\right)^{p / 2}\right)^{1 / p} \leq \sqrt{d}\left(\sum_{n=1}^{d}\left(\mathbf{E}\left(\int_{0}^{t} Y_{n}^{2}(u) \mathrm{d} u\right)^{p / 2}\right)^{2 / p}\right)^{1 / 2} \\
& \leq \sqrt{d}\left(\int_{0}^{t} \sum_{n=1}^{d}\left(\mathbf{E}\left|Y_{n}(u)\right|^{p}\right)^{2 / p} \mathrm{~d} u\right)^{1 / 2} \leq \sqrt{d}\left(\int_{0}^{t} d^{1-\frac{2}{p}}\left(\mathbf{E} \sum_{n=1}^{d}\left|Y_{n}(u)\right|^{p}\right)^{2 / p} \mathrm{~d} u\right)^{1 / 2} \\
& \leq d^{1-1 / p}\left(\int_{0}^{t}\left(\mathbf{E}\left(\sum_{n=1}^{d} Y_{n}^{2}(u)\right)^{p / 2}\right)^{2 / p} \mathrm{~d} u\right)^{1 / 2} \leq d^{1-1 / p}\left(\int_{0}^{t} f_{p}^{2}(u) \mathrm{d} u\right)^{1 / 2} .
\end{aligned}
$$

The term (3.9) can be estimated similarly:

$$
\begin{aligned}
\sum_{n=1}^{d} & \left(\mathbf{E}\left(\int_{0}^{t}\left|Y_{n}(u)\right| \mathrm{d} u\right)^{p}\right)^{1 / p} \leq \int_{0}^{t} \sum_{n=1}^{d}\left(\mathbf{E}\left|Y_{n}(u)\right|^{p}\right)^{1 / p} \mathrm{~d} u \\
& \leq d^{1-1 / p} \int_{0}^{t}\left(\mathbf{E} \sum_{n=1}^{d}\left|Y_{n}(u)\right|^{p}\right)^{1 / p} \mathrm{~d} u \leq d^{1-1 / p} \int_{0}^{t}\left(\mathbf{E}\left(\sum_{n=1}^{d} Y_{n}^{2}(u)\right)^{p / 2}\right)^{1 / p} \mathrm{~d} u \\
& \leq d^{1-1 / p}\left(\int_{0}^{t} f_{p}(u) \mathrm{d} u\right) .
\end{aligned}
$$


Therefore we obtain

$$
\begin{aligned}
f_{p}(t) & =\max _{i}\left(\mathbf{E} Z^{p}(t)\right)^{1 / p} \leq \max _{i} \sum_{j=1}^{d}\left(\mathbf{E} \sup _{0 \leq s \leq t}\left|Y_{j}(s)\right|^{p}\right)^{1 / p} \\
& \leq 1+d^{2-1 / p} \sqrt{k_{1}} C_{p}^{1 / p}\left(\int_{0}^{t} f_{p}^{2}(s) \mathrm{d} s\right)^{1 / 2}+d^{2-1 / p} k_{3} \int_{0}^{t} f_{p}(s) \mathrm{d} s .
\end{aligned}
$$

Taking squares and using the formula $(A+B+C)^{2} \leq \alpha_{1} A^{2}+\alpha_{2} B^{2}+\alpha_{3} C^{2}$ for $A, B, C \geq 0$, we obtain

$$
f_{p}^{2}(t) \leq \alpha_{1}+\alpha_{2} d^{4-2 / p} k_{1} C_{p}^{2 / p} \int_{0}^{t} f_{p}^{2}(s) \mathrm{d} s+\alpha_{3} d^{4-2 / p} k_{3}^{2}\left(\int_{0}^{t} f_{p}(s) \mathrm{d} s\right)^{2},
$$

and hence (3.7) by Lemma 4.3

Let us now treat the two-point differences and recall from [6, p. 123] that

$V_{j}(t)=\sum_{n=1}^{d}\left(\int_{0}^{t} V_{n}(s) D_{n} F_{j}\left(x_{s}, \mathrm{~d} s\right)+\int_{0}^{t} D_{i} \phi_{s}^{n}(y)\left(D_{n} F_{j}\left(x_{s}, \mathrm{~d} s\right)-D_{n} F_{j}\left(y_{s}, \mathrm{~d} s\right)\right)\right)$.

Then for $p \geq 2$ we have

$$
\begin{aligned}
\left(\mathbf{E}\left(\sum_{j=1}^{d} \sup _{0 \leq t \leq T} V_{j}^{2}(t)\right)^{p / 2}\right)^{2 / p} \leq \sum_{j=1}^{d}\left(\mathbf{E} \sup _{0 \leq t \leq T} V_{j}^{p}(t)\right)^{2 / p} \\
\leq \sum_{j=1}^{d}\left(\sum_{n=1}^{d}\left(\mathbf{E} \sup _{0 \leq t \leq T}\left|\int_{0}^{t} V_{n}(s) D_{n} M_{j}\left(x_{s}, \mathrm{~d} s\right)\right|^{p}\right)^{1 / p}\right. \\
\quad+\sum_{n=1}^{d}\left(\mathbf{E} \sup _{0 \leq t \leq T}\left|\int_{0}^{t} D_{i} \phi_{s}^{n}(y)\left(D_{n} M_{j}\left(x_{s}, \mathrm{~d} s\right)-D_{n} M_{j}\left(y_{s}, \mathrm{~d} s\right)\right)\right|^{p}\right)^{1 / p} \\
\quad+\sum_{n=1}^{d}\left(\mathbf{E} \sup _{0 \leq t \leq T}\left|\int_{0}^{t} V_{n}(s) D_{n} b_{j}\left(x_{s}, s\right) \mathrm{d} s\right|^{p}\right)^{1 / p} \\
\left.\left.\quad+\left.\sum_{n=1}^{d}\left(\mathbf{E} \sup _{0 \leq t \leq T} \mid \int_{0}^{t} D_{i} \phi_{s}^{n}(y)\left(D_{n} b_{j}\left(x_{s}, s\right) \mathrm{d} s-D_{n} b_{j}\left(y_{s}, s\right)\right) \mathrm{d} s\right)\right|^{p}\right)^{1 / p}\right)^{2} .
\end{aligned}
$$

We have by Burkholder's inequality

$$
\begin{aligned}
\mathbf{E} \sup _{0 \leq t \leq T}\left|\int_{0}^{t} V_{n}(s) D_{n} M_{j}\left(x_{s}, \mathrm{~d} s\right)\right|^{p} & \leq C_{p} \mathbf{E}\left(\left|\int_{0}^{T} V_{n}^{2}(t) D_{n} D_{n} a_{j j}\left(x_{t}, x_{t}, t\right) \mathrm{d} t\right|^{p / 2}\right) \\
& \leq C_{p} k_{1}^{p / 2} \mathbf{E}\left(\left|\int_{0}^{T} V_{n}^{2}(t) \mathrm{d} t\right|^{p / 2}\right)
\end{aligned}
$$


and

$$
\begin{aligned}
& \left(\mathbf{E} \sup _{0 \leq t \leq T}\left|\int_{0}^{t} D_{i} \phi_{s}^{n}(y)\left(D_{n} M_{j}\left(x_{s}, \mathrm{~d} s\right)-D_{n} M_{j}\left(y_{s}, \mathrm{~d} s\right)\right)\right|^{p}\right)^{1 / p} \\
& \leq C_{p}^{1 / p}\left(\mathbf{E}\left|\int_{0}^{T}\left(D_{i} \phi_{s}^{n}(y)\right)^{2} D_{n} D_{n} \mathcal{A}_{j j}\left(x_{s}, y_{s}, s\right) \mathrm{d} s\right|^{p / 2}\right)^{1 / p} \\
& \leq C_{p}^{1 / p} k_{2}^{1 / 2}\left(\mathbf{E}\left|\int_{0}^{T}\left(D_{i} \phi_{s}^{n}(y)\right)^{2}\right| \phi_{s}(x)-\left.\left.\phi_{s}(y)\right|^{2} \mathrm{~d} s\right|^{p / 2}\right)^{1 / p} \\
& \leq C_{p}^{1 / p} k_{2}^{1 / 2}\left|\int_{0}^{T}\left(\mathbf{E}\left|D_{i} \phi_{s}^{n}(y)\right|^{p}\left|\phi_{s}(x)-\phi_{s}(y)\right|^{p}\right)^{2 / p} \mathrm{~d} s\right|^{1 / 2} \\
& \leq C_{p}^{1 / p} k_{2}^{1 / 2}\left|\int_{0}^{T}\left(\mathbf{E}\left|D_{i} \phi_{s}^{n}(y)\right|^{2 p}\right)^{1 / p}\left(\mathbf{E}\left|\phi_{s}(x)-\phi_{s}(y)\right|^{2 p}\right)^{1 / p} \mathrm{~d} s\right|^{1 / 2} \\
& \leq C_{p}^{1 / p} k_{2}^{1 / 2}\left|\int_{0}^{T} \tilde{f}_{2 p}^{2}(s) \bar{c}^{2}\right| x-\left.\left.y\right|^{2} \mathrm{e}^{2\left(\Lambda+p \sigma^{2}\right) s} \mathrm{~d} s\right|^{1 / 2}
\end{aligned}
$$

and

$$
\mathbf{E} \sup _{0 \leq t \leq T}\left|\int_{0}^{t} V_{n}(s) D_{n} b_{j}\left(x_{s}, s\right) \mathrm{d} s\right|^{p} \leq k_{3}^{p} \mathbf{E}\left(\int_{0}^{T}\left|V_{n}(s)\right| \mathrm{d} s\right)^{p}
$$

and

$$
\begin{aligned}
& \left(\mathbf{E} \sup _{0 \leq t \leq T}\left|\int_{0}^{t} D_{i} \phi_{s}^{n}(y)\left(D_{n} b_{j}\left(x_{s}, s\right)-D_{n} b_{j}\left(y_{s}, s\right)\right) \mathrm{d} s\right|^{p}\right)^{1 / p} \\
\leq & k_{4}\left(\mathbf{E}\left(\int_{0}^{T}\left|D_{i} \phi_{s}^{n}(y)\right|\left|\phi_{s}(x)-\phi_{s}(y)\right| \mathrm{d} s\right)^{p}\right)^{1 / p} \leq k_{4} \bar{c}|x-y| \int_{0}^{T} \tilde{f}_{2 p}(s) \mathrm{e}^{\left(\Lambda+\sigma^{2} p\right) s} \mathrm{~d} s .
\end{aligned}
$$

Therefore, using the same estimates as in the first part of the proof, we get

$$
\begin{aligned}
g_{p}^{2}(T) \leq & d\left(d^{1-1 / p} C_{p}^{1 / p} k_{1}^{1 / 2}\left(\int_{0}^{T} g_{p}^{2}(t) \mathrm{d} t\right)^{1 / 2}\right. \\
& +d C_{p}^{1 / p} \bar{c}|x-y| k_{2}^{1 / 2}\left(\int_{0}^{T} \tilde{f}_{2 p}^{2}(s) \mathrm{e}^{2\left(\Lambda+p \sigma^{2}\right) s} \mathrm{~d} s\right)^{1 / 2} \\
& \left.+d^{1-1 / p} k_{3} \int_{0}^{T} g_{p}(s) \mathrm{d} s+d k_{4} \bar{c}|x-y| \int_{0}^{T} \tilde{f}_{2 p}(s) \mathrm{e}^{\left(\Lambda+\sigma^{2} p\right) s} \mathrm{~d} s\right)^{2} \\
& \leq \beta_{1} d^{3-2 / p} C_{p}^{2 / p} k_{1} \int_{0}^{T} g_{p}^{2}(t) \mathrm{d} t+\beta_{3} d^{3-2 / p} k_{3}^{2}\left(\int_{0}^{T} g_{p}(t) \mathrm{d} t\right)^{2}+H(T),
\end{aligned}
$$

where $H$ is as in the lemma. Therefore, (3.8) follows from Lemma4.3 and the proof is complete. 
Theorem 3.2. Let $d \geq 2$ and let $\phi$ be a stochastic flow satisfying the assumptions of Lemma 3.1 For any (deterministic, compact) subset $\mathbb{X}$ of $\mathbf{R}^{d}$ with box dimension $\Delta \geq 0$, we have

$$
\limsup _{T \rightarrow \infty} \frac{1}{T} \log \sup _{x \in \mathbb{X}} \sup _{0 \leq t \leq T}\left\|D \phi_{0, t}(x)\right\| \leq \xi
$$

where

$$
\xi= \begin{cases}\hat{k} & \text { if } \hat{k} \geq c d+\hat{c} \\ \hat{k}+2 \sqrt{k \Delta \gamma_{1}} & \text { if } \hat{k} \leq c d+\hat{c} \text { and } 2 \sqrt{c k} \Delta d+c d^{2}-2 c \Delta d+\Delta(\hat{k}-\hat{c}) \geq 0 \\ \hat{k}+2 \sqrt{k \Delta \gamma_{2}} & \text { if } \hat{k} \leq c d+\hat{c} \text { and } 2 \sqrt{c k} \Delta d+c d^{2}-2 c \Delta d+\Delta(\hat{k}-\hat{c}) \leq 0,\end{cases}
$$

where $\gamma_{1}$ and $\gamma_{2}$ are defined as in Theorem 2.2 and

$$
\begin{aligned}
& k=\alpha_{2} d^{4} k_{1} k_{5}^{2} / 2, \quad \hat{k}=\sqrt{\alpha_{3}} d^{2} k_{3}+2 k \\
& c=\alpha_{2} d^{4} k_{1} k_{5}^{2}+\frac{1}{2} \beta_{1} d^{3} k_{1} k_{5}^{2}+\sigma^{2}, \quad \hat{c}=\sqrt{\alpha_{3}} d^{2} k_{3}+\sqrt{\beta_{3} d^{3} k_{3}^{2}}+\Lambda .
\end{aligned}
$$

Proof. The proof is just a combination of Lemma 3.1 and Theorem 2.2 Lemma 3.1 tells us that

$$
\left(\mathbf{E} \sup _{0 \leq t \leq T}\left\|D \phi_{t}(x)\right\|^{p}\right)^{1 / p} \leq \exp \{(k p+\tilde{k}) T+o(T)\}
$$

for all $p \geq 2$, where

$$
k=\alpha_{2} d^{4} k_{1} k_{5}^{2} / 2, \quad \tilde{k}=\sqrt{\alpha_{3}} d^{2} k_{3} .
$$

In order to obtain an estimate for all $p \geq 0$, we define $\hat{k}:=2 k+\tilde{k}$ and get

$$
\left(\mathbf{E} \sup _{0 \leq t \leq T}\left\|D \phi_{t}(x)\right\|^{p}\right)^{1 / p} \leq \exp \{(k p+\hat{k}) T+o(T)\}
$$

for all $p \geq 0$.

Lemma 3.1 tells us further that for $p \geq 2$ (and hence for $p>d$ )

$$
\mathbf{E} \sup _{0 \leq t \leq T}\left\|D \phi_{t}(x)-D \phi_{t}(y)\right\|^{p} \leq|x-y|^{p} \exp \left\{\left(c p^{2}+\hat{c} p\right) T+o(T)\right\},
$$

with $c, \bar{c}$ as in the theorem. Therefore, the assumptions of Theorem 2.2 hold and the assertion follows.

Remark 3.3. The formulas in Theorem 3.2 still contain the numbers $\alpha_{2}, \alpha_{3}, \beta_{1}$ and $\beta_{3}$. Since $\alpha_{1}, \beta_{2}$ and $\beta_{4}$ do not appear in the formulas, it is possible to choose $\alpha_{2}=$ $\alpha_{3}=\beta_{1}=\beta_{3}=2$ but a different choice may result in a sharper bound.

Remark 3.4. One can establish a corresponding result also in case $d=1$ by adjusting $\hat{c}$ just like we adjusted $\hat{k}$ in order to extend the moment bound on the differences of derivatives from $p \geq 2$ to $p>d=1$.

Remark 3.5. It is not hard to establish a version of both Lemma 3.1 and Theorem 3.2 for higher derivatives of a stochastic flow using an induction proof along the lines of $[6$, Proposition 2.3]. 


\section{Isotropic Brownian Flows}

In this section we will specialize the results of Theorem 3.2 to isotropic Brownian flows (IBFs). We will be able to establish somewhat better upper bounds by exploiting - for example - an explicit representation of the growth of the derivative of an IBF. We start by defining an IBF. We assume that $d \geq 2$.

Definition 4.1. A stochastic flow $\phi$ on $\mathbf{R}^{d}$ is called isotropic Brownian flow if it is generated by a semimartingale field $F=M+V$ with $V \equiv 0$ and martingale field $M$ with quadratic variation $a(x, y, t, \omega)=b(x-y)$, where $b: \mathbf{R}^{d} \rightarrow \mathbf{R}^{d \times d}$ is a deterministic function satisfying

- $x \mapsto b(x)$ is $C^{4}$.

- $b(0)=\mathrm{id}_{\mathbf{R}^{d}}$.

- $x \mapsto b(x)$ is not constant.

- $b(x)=O^{*} b(O x) O$ for any $x \in \mathbf{R}^{d}$ and any orthogonal matrix $O \in O(d)$.

For an IBF, we define its longitudinal resp. normal correlation functions by

$$
B_{L}(r):=b_{11}\left(r e_{1}\right), \quad B_{N}(r):=b_{11}\left(r e_{2}\right), \quad r \geq 0
$$

where $e_{i}$ denotes the $i^{\text {th }}$ unit vector in $\mathbf{R}^{d}$ ( 1 and 2 can be replaced by any $i \neq j$ by isotropy).

We will need the following facts about IBFs.

- $\beta_{L}:=-B_{L}^{\prime \prime}(0)>0, \beta_{N}:=-B_{N}^{\prime \prime}(0)>0$.

- For each $x \in \mathbf{R}^{d}$, we have $\lambda_{1}:=\frac{1}{2}\left((d-1) \beta_{N}-\beta_{L}\right)=\lim _{t \rightarrow \infty} \frac{1}{t} \log \left\|D \phi_{t}(x)\right\|$ almost surely. The number $\lambda_{1}$ is called the top Lyapunov exponent of the IBF.

- $b, B_{L}$ and $B_{N}$ are bounded with bounded derivatives up to order 2 .

- $k_{1}:=\max _{i, j}\left|D_{x_{i}} D_{y_{i}} b_{j j}(x-y)\right|_{x=y}=\beta_{L} \vee \beta_{N}$ (independently of $x$ )

- $F$ satisfies Hypothesis (A).

The first two of these facts can be found in [2], $b$ is bounded since $b$ is a covariance function and boundedness of the second derivatives (and therefore also of the first) follows from equation (4.10). The fourth item follows from the definition of $\beta_{L}$ and $\beta_{N}$ and the final one from the boundedness of the second derivatives of $b$.

Theorem 4.1. Let $d \geq 2$ and let $\phi$ be an IBF. For any (deterministic, compact) subset $\mathbb{X}$ of $\mathbf{R}^{d}$ with box dimension $\Delta \geq 0$, we have

$$
\limsup _{T \rightarrow \infty} \frac{1}{T} \log \sup _{x \in \mathbb{X}} \sup _{0 \leq t \leq T}\left\|D \phi_{0, t}(x)\right\| \leq \xi
$$

where $\xi$ is defined as in Theorem 2.2 with

$$
c=2 \beta_{L}+10 d^{3}\left(\beta_{L} \vee \beta_{N}\right), \quad \hat{c}=2 \lambda_{1}, \quad k=\frac{\beta_{L}}{2}, \quad \hat{k}=\lambda_{1}^{+} .
$$


Proof. Defining $f_{p}$ and $\tilde{f}_{p}$ as in the previous section, we obtain by Lemma 4.6 .

$$
f_{p}(t) \leq \exp \left\{\left(\lambda_{1}^{+}+\frac{\beta_{L}}{2} p\right) t+o(t)\right\}, \quad \tilde{f}_{p}(t) \leq \exp \left\{\left(\lambda_{1}+\frac{\beta_{L}}{2} p\right) t+o(t)\right\}
$$

for all $p>0$. Next, we estimate $g_{p}$ according to formula (3.8). Observing that $\bar{C}_{2}=0$, $\Lambda=\lambda_{1}$ and $\sigma=\sqrt{\beta_{L}}$ (by Lemma4.5), we obtain

$$
g_{p}(t) \leq|x-y| \exp \left\{\left(2 \lambda_{1}+2 \beta_{L} p+\frac{1}{2} d^{3}\left(\beta_{L} \vee \beta_{N}\right) C_{p}^{2 / p}\right) t+o(t)\right\} .
$$

Noting that $C_{p}^{1 / p} \leq 2 \sqrt{5} p^{1 / 2}$ for $p \geq 2$ [1, Proposition 4.2], the assertion follows from Theorem 2.2.

Remark 4.2. Even though we have no reason to believe that the bound in Theorem 4.1 is optimal in general, it is at least optimal in case $\Delta=0$ (by Lemma 4.6).

\section{Appendix A: A Gronwall-type Lemma}

We include in this section an elementary lemma which we conjecture to be essentially well-known but which we could not find in the literature in a version suitable for our needs.

Lemma 4.3. Let $f:[0, \infty) \rightarrow[0, \infty)$ be a locally integrable function and $H$ : $[0, \infty) \rightarrow[0, \infty)$ non-decreasing such that

$$
f(t) \leq C_{1} \int_{0}^{t} f(s) \mathrm{d} s+C_{2}\left(\int_{0}^{t} \sqrt{f(s)} \mathrm{d} s\right)^{2}+H(t)
$$

for some $C_{1}, C_{2} \geq 0$ and all $t \geq 0$. Then

$$
f(t) \leq H(t)+H(t) \frac{C_{1}+\sqrt{C_{2}}}{C_{1}+2 \sqrt{C_{2}}}\left(\exp \left\{\left(C_{1}+2 \sqrt{C_{2}}\right) t\right\}-1\right)
$$

for all $t \geq 0$.

Proof. Let $\lambda>0$ and $\gamma_{t}:=\lambda\left(1-\mathrm{e}^{-\lambda t}\right)^{-1}$ for $t>0$. Then, by Jensen's inequality, for $t>0$

$$
\begin{aligned}
f(t) & \leq C_{1} \int_{0}^{t} f(s) \mathrm{d} s+C_{2}\left(\int_{0}^{t} \sqrt{f(s)} \frac{\mathrm{e}^{\lambda(t-s)}}{\gamma_{t}} \gamma_{t} \mathrm{e}^{-\lambda(t-s)} \mathrm{d} s\right)^{2}+H(t) \\
& \leq C_{1} \int_{0}^{t} f(s) \mathrm{d} s+C_{2} \int_{0}^{t} f(s) \frac{\mathrm{e}^{\lambda(t-s)}}{\gamma_{t}} \mathrm{~d} s+H(t) .
\end{aligned}
$$

Therefore,

$$
\begin{aligned}
\mathrm{e}^{-\lambda t} f(t) & \leq C_{1} \mathrm{e}^{-\lambda t} \int_{0}^{t} f(s) \mathrm{d} s+\frac{C_{2}}{\lambda} \int_{0}^{t} f(s) \mathrm{e}^{-\lambda s} \mathrm{~d} s+H(t) \mathrm{e}^{-\lambda t} \\
& \leq C_{1} \int_{0}^{t} \mathrm{e}^{-\lambda s} f(s) \mathrm{d} s+\frac{C_{2}}{\lambda} \int_{0}^{t} f(s) \mathrm{e}^{-\lambda s} \mathrm{~d} s+H(t) \mathrm{e}^{-\lambda t} .
\end{aligned}
$$


Gronwall's Lemma implies

$$
\begin{aligned}
f(t) & \leq \mathrm{e}^{\lambda t}\left(H(t) \mathrm{e}^{-\lambda t}+\int_{0}^{t} H(s) \mathrm{e}^{-\lambda s}\left(C_{1}+\frac{C_{2}}{\lambda}\right) \mathrm{e}^{\left(C_{1}+\frac{C_{2}}{\lambda}\right)(t-s)} \mathrm{d} s\right) \\
& =H(t)+\mathrm{e}^{\lambda t} \int_{0}^{t} H(s) \mathrm{e}^{-\lambda s}\left(C_{1}+\frac{C_{2}}{\lambda}\right) \mathrm{e}^{\left(C_{1}+\frac{C_{2}}{\lambda}\right)(t-s)} \mathrm{d} s .
\end{aligned}
$$

Choosing $\lambda=\sqrt{C_{2}}$ (which minimizes $\lambda+C_{1}+\frac{C_{2}}{\lambda}$ ) and using the monotonicity of $H$, we obtain

$$
f(t) \leq H(t)+H(t) \frac{C_{1}+\sqrt{C_{2}}}{C_{1}+2 \sqrt{C_{2}}}\left(\mathrm{e}^{\left(C_{1}+2 \sqrt{C_{2}}\right) t}-1\right)
$$

as claimed in the lemma.

\section{Appendix B: Some Estimates for IBFs}

In this appendix, we collect three basic properties of IBFs which are used in Section 4 and which do not seem to have appeared in the literature so far.

Lemma 4.4. Let $\phi$ be an IBF with covariance tensor $b$. Then we obtain for the correlation functions $B_{N / L}$ defined in Section 4

$$
1-B_{L}(r) \leq \frac{\beta_{L}}{2} r^{2} \text { and } 1-B_{N}(r) \leq \frac{\beta_{N}}{2} r^{2}
$$

for all $r \geq 0$.

Proof. Let $U(x), x \in \mathbf{R}^{d}$ be an $\mathbf{R}^{d}$-valued centered Gaussian process with $\operatorname{cov}\left(U_{i}(x), U_{j}(y)\right)=$ $b_{i j}(x-y)$. Denoting the $i^{\text {th }}$ unit coordinate vector by $\mathrm{e}_{i}$ and using Schwarz' inequality, we get

$$
\begin{aligned}
B_{L}^{\prime \prime}(r) & =\lim _{h \rightarrow 0} \lim _{\delta \rightarrow 0} \mathbf{E}\left(\frac{U_{1}\left(h \mathrm{e}_{1}\right)-U_{1}(0)}{h} \frac{U_{1}\left(-(r+\delta) \mathrm{e}_{1}\right)-U\left(-r \mathrm{e}_{1}\right)}{\delta}\right) \\
& =-\mathbf{E}\left(U_{1}^{\prime}\left(r \mathrm{e}_{1}\right) U_{1}^{\prime}(0)\right) \geq-\mathbf{E}\left(U_{1}^{\prime}(0)^{2}\right)=B_{L}^{\prime \prime}(0)
\end{aligned}
$$

Therefore, for each $r>0$ there exists some $\theta \in(0, r)$ such that

$$
B_{L}(r)=B_{L}(0)+\frac{1}{2} B_{L}^{\prime \prime}(\theta) r^{2} \geq 1+\frac{1}{2} B_{L}^{\prime \prime}(0) r^{2}=1-\frac{1}{2} \beta_{L} r^{2}
$$

The estimate for $B_{N}$ follows in the same way, so the assertion of the lemma follows.

Observe that the following lemma holds for every IBF - even if the top exponent $\lambda_{1}$ is negative. 
Lemma 4.5. Let $\phi$ be an IBF. Let $x, y \in \mathbf{R}^{d}, x \neq y$ and let $\rho(t):=\left|\phi_{t}(x)-\phi_{t}(y)\right|$.

Then

$$
\mathbf{E} \rho_{t}^{q} \leq|x-y|^{q} \exp \left\{\left(q \lambda_{1}+q^{2} \frac{\beta_{L}}{2}\right) t\right\}
$$

for all $t \geq 0$ and all $q \geq 1$.

Proof. We know that

$$
\mathrm{d} \rho_{t}=(d-1) \frac{1-B_{N}\left(\rho_{t}\right)}{\rho_{t}} \mathrm{~d} t+\sqrt{2\left(1-B_{L}\left(\rho_{t}\right)\right)} \mathrm{d} W_{t} .
$$

Let $q \geq 1$. Itô's formula implies

$$
\begin{aligned}
\mathrm{d} \rho_{t}^{q} & =q \rho_{t}^{q-1} \mathrm{~d} \rho_{t}+\frac{q(q-1)}{2} \rho_{t}^{q-2} \mathrm{~d}\langle\rho\rangle_{t} \\
& =q \rho_{t}^{q-2}\left((d-1)\left(1-B_{N}\left(\rho_{t}\right)\right)+(q-1)\left(1-B_{L}\left(\rho_{t}\right)\right)\right) \mathrm{d} t+q \rho_{t}^{q-1} \sqrt{2\left(1-B_{L}\left(\rho_{t}\right)\right)} \mathrm{d} W_{t} .
\end{aligned}
$$

By Lemma 4.4, we get

$$
\mathbf{E} \rho_{t}^{q} \leq|x-y|^{q}+q\left(\frac{\beta_{N}}{2}(d-1)+\frac{\beta_{L}}{2}(q-1)\right) \int_{0}^{t} \mathbf{E} \rho_{s}^{q} \mathrm{~d} s .
$$

Gronwall's Lemma, together with the formula for the top exponent $\lambda_{1}=(d-1) \beta_{N} / 2-$ $\beta_{L} / 2$ imply the assertion.

Next, we look at the derivative of an IBF. The formula in the next lemma becomes particularly nice if we use the following Schatten norm of a $d \times d$-matrix $A=\left(a_{i j}\right)$ :

$$
\|A\|_{S}:=\left(\sum_{i, j=1}^{d}\left(\sum_{k=1}^{d} a_{i k} a_{j k}\right)^{2}\right)^{1 / 4}=\left(\sum_{i=1}^{d} \sigma_{i}^{4}\right)^{1 / 4},
$$

where $\sigma_{1}, \ldots, \sigma_{d}$ are the singular values of $A$.

Lemma 4.6. Let $\phi$ be an IBF. Then, for each $x \in \mathbf{R}^{d}$, there exists a one-dimensional Wiener process $W$ such that for all $t \geq 0$,

$$
\left\|D \phi_{t}(x)\right\|_{S}=d^{1 / 4} \exp \left\{\lambda_{1} t+\sqrt{\beta_{L}} W_{t}\right\} .
$$

Proof. Fix $x \in \mathbf{R}^{d}$ and define

$$
N_{t}:=\int_{0}^{t} \sum_{i, j, k} D_{k} M^{i}\left(\phi_{s}(x), \mathrm{d} s\right) \frac{D_{j} \phi_{s}^{j}(x) D_{j} \phi_{s}^{k}(x)}{\left\|D \phi_{s}(x)\right\|_{S}} .
$$

Then it is easy to see (cf. [14, p. 101]) that

$$
\langle N\rangle_{t}=\beta_{L} t .
$$

Therefore, $W_{t}:=\left(\beta_{L}\right)^{-1 / 2} N_{t}, t \geq 0$ is a standard Brownian motion and applying Itô's formula, we get

$$
\log \left\|D \phi_{t}(x)\right\|_{S}=\frac{1}{4} \log d+N_{t}+\lambda_{1} t=\frac{1}{4} \log d+\sqrt{\beta_{L}} W_{t}+\lambda_{1} t .
$$

Exponentiating this expression, the lemma follows. 


\section{References}

[1] M. Barlow and M. Yor. Semi-martingale inequalities via the Garsia-RodemichRumsey Lemma, and applications to local times. J. Func. Anal., 49:198-229, 1982.

[2] P. Baxendale and T. Harris. Isotropic stochastic flows. Ann. Probab., 14(2):1155$1179,1986$.

[3] M. Cranston, M. Scheutzow, and D. Steinsaltz. Linear expansion of isotropic Brownian flows. Elect. Comm. in Probab., 4:91-101, 1999.

[4] M. Cranston, M. Scheutzow, and D. Steinsaltz. Linear bounds for stochastic dispersion. Ann. Probab., 28(4):1852-1869, 2000.

[5] D. Dolgopyat, V. Kaloshin, and L. Koralov. A limit shape theorem for periodic stochastic dispersion. Comm. Pure Appl. Math., 57(9):1127-1158, 2004.

[6] P. Imkeller and M. Scheutzow. On the spatial asymptotic behaviour of stochastic flows in Euclidean space. Ann. Probab., 27:109-129, 1999.

[7] H. Kunita. Stochastic Flows and Stochastic Differential Equations. Cambridge University Press, Cambridge, UK, 1990.

[8] H. Lisei and M. Scheutzow. Linear bounds and Gaussian tails in a stochastic dispersion model. Stochastics and Dynamics, 1(3):389-403, 2001.

[9] H. Lisei and M. Scheutzow. On the dispersion of sets under the action of an isotropic Brownian flow. In Probabilistic Methods in Fluids, pages 224-238. World Scientific, Singapore, 2003.

[10] P.-D. Liu and M. Qian. Smooth ergodic theory of random dynamical systems, volume 1606 of Lecture Notes In Mathematics. Springer, 1995.

[11] M. Scheutzow. Chaining techniques and their application to stochastic flows. In J. Blath, P. Mörters, and M. Scheutzow, editors, Trends in Stochastic Analysis, volume 353 of LMS Lecture Note Series, pages 35-63. Cambridge University Press, Cambridge, UK, 2009.

[12] M. Scheutzow and D. Steinsaltz. Chasing balls through martingale fields. Ann. Probab., 30(4):2046-2080, 2002.

[13] H. van Bargen. Asymptotic growth of spatial derivatives of isotropic flows. Electron. J. Probab., 14:2328-2351, 2009.

[14] H. van Bargen. Some Asymptotic Properties of Stochastic Flows. PhD thesis, TU Berlin, $2010 . \quad$ URL: http://opus.kobv.de/tuberlin/volltexte/2010/2571/pdf/vanbargen_holger.pdf. 